\title{
Estabilidad de los puntos de críticos de los sistemas dinámicos autónomos lineales
}

\section{Stability of the equilibrium points of linear autonomous dynamic systems}

\author{
${ }^{1}$ Jhony Alfonso Chávez Delgado \\ ${ }^{2}$ Eduardo Rodríguez Delgado \\ ${ }^{3}$ Luis César Méndez Avalos \\ ${ }^{4}$ Jeaneth Gaby Atahuachi Catacora
}

\author{
ORCID: 0000-0001-6512-2285 \\ ORCID: 0000-0001-5975-8236 \\ ORCID: 0000-0002-6059-1434 \\ ORCID: 0000-0001-5074-3185
}

\section{RESUMEN}

Esta investigación tuvo como objetivo establecer la estabilidad y la estabilidad asintótica sin conocer la solución del sistema dinámico autónomo lineal, la cual puede resultar difícil, incluso, imposible. Se empleó el método inductivo y deductivo para establecer un criterio de estabilidad a través de la solución constante de un sistema autónomo lineal. Como resultado, se generalizó la estabilidad de los puntos críticos de manera cuantitativa, examinando los valores propios de una matriz cuadrada no singular. Esta matriz tiene la propiedad de que el sistema autónomo es asintóticamente estable si las partes reales de todos los valores propios de la matriz no singular son negativos, y estable si la matriz no singular tiene un par de valores imaginarios puros de multiplicidad uno. Así mismo, se clasificaron los puntos de críticos cualitativamente en el plano de fase. La estabilidad se justifica principalmente por la importancia que tiene en la moderna teoría de control y en la estabilidad de los sistemas dinámicos no lineales.

Palabras clave: Estabilidad, estabilidad asintótica, sistema dinámico autónomo no lineal.

\begin{abstract}
The purpose of this article is to establish stability and asymptotic stability without knowing the linear autonomous dynamic system solution, which could be difficult to impose. The inductive and deductive method was used to establish a stability criterion through the constant solution of a linear autonomous system. As a result, the stability of the critical points was generalized, quantitatively, examining the eigenvalues of a non-singular square matrix. This matrix has the property that the autonomous system is asymptotically stable if the real parts of all the eigenvalues of the non-singular matrix are negative, and stable if the non-singular matrix has a pair of pure imaginary values of multiplicity one. Likewise, the critical points were qualitatively classified in the phase plane. Stability is justified mainly by its importance in modern control theory and in the stability of nonlinear dynamical systems.
\end{abstract}

Keywords: Stability, asymptotic stability, linear autonomous dynamic system.

\footnotetext{
${ }^{1}$ Universidad Nacional Jorge Basadre Grohmann. Tacna, Perú. E-mail: jchavezd@ unjbg.edu.pe

${ }^{2}$ Universidad Nacional Jorge Basadre Grohmann. Tacna, Perú. E-mail: erodriguezd@ unjbg.edu.pe

${ }^{3}$ Universidad Nacional Jorge Basadre Grohmann. Tacna, Perú. E-mail: 1mendeza @unjbg.edu.pe

${ }^{4}$ Universidad Nacional Jorge Basadre Grohmann. Tacna, Perú. E-mail:jatahuachic@unjbg.edu.pe
} 


\section{INTRODUCCIÓN}

El problema de la estabilidad surgió en la ingeniería de control, la máquina de vapor de James Watts sobre la velocidad de las máquinas que variaban cíclicamente en el tiempo con comportamiento no muy estables, o el diseño de control de George Airy para la regulación de la velocidad telescópica. También, Maxwell demostró que el comportamiento de un sistema de control automático se relacionaba con las raíces del polinomio característico (Maxwell, 1868).

La contribución más importante en el siglo XIX se debe a Henry Poincaré, quien aplicó la topología para estudiar las propiedades de las trayectorias. La teoría cualitativa de las ecuaciones diferenciales fue creada simultáneamente por Poincaré y Liapunov. Henry Poincaré pensaba que para saber si el sistema solar es estable y conocer si un cuerpo permanece en un lugar del espacio o se aleja, no tiene sentido iniciar el estudio cuantitativo si no se conoce antes el comportamiento de las soluciones, por lo que hace falta un estudio cualitativo. Poincaré se propuso a averiguar el comportamiento de las soluciones de una ecuación diferencial o de un sistema de dos ecuaciones en todo el plano, y hacerlo sin integrar las ecuaciones, utilizando únicamente las propiedades de las funciones (Poincaré, Russell, Zermelo et Peano, 1986).

Las investigaciones sobre las ecuaciones diferenciales ordinarias en un principio fueron tratadas por métodos analíticos estrictos, en la actualidad tiene un tratamiento moderno mediante los sistemas dinámicos.

Un sistema dinámico autónomo es un sistema de ecuaciones diferenciales ordinarias de primer orden, con campo vectorial estacionario o independiente del tiempo, y se representan por un modelo matemático:

$$
\dot{\overline{\mathrm{x}}}=\mathrm{f}(\overline{\mathrm{x}})
$$

, donde $\mathrm{f}: \mathrm{U} \rightarrow \mathbb{R}^{\mathrm{n}}$ es una aplicación diferenciable continua definida en un conjunto abierto $\mathrm{U} \subset \mathbb{R}^{\mathrm{n}}$.

En el análisis del sistema dinámico autónomo $(1,1)$, se limita a investigar tres aspectos fundamentales: Existencia, unicidad y estabilidad de la solución constante, denominada puntos críticos o de equilibrio.

De especial interés son los puntos críticos, que son soluciones que no dependen del tiempo, es decir, la función constante es idénticamente igual al punto crítico del sistema autónomo y su trayectoria asociada se reducirá a un punto. Esto implica que el punto crítico se anula en el campo vectorial (Chávez, 2018).

Como el punto crítico se anula en el campo vectorial, el problema de la estabilidad y estabilidad asintótica, consiste en determinar las condiciones bajo las cuales aquellas soluciones del sistema dinámico autónomo, que se originan cercan de un punto crítico de este sistema dinámico permanecen cerca del punto, en tal caso decimos, que el punto crítico es estable. Si además existiera la posibilidad de acercarse al punto crítico como una posición límite, entonces el punto crítico es asintóticamente estable. En caso contrario se dice que el punto crítico es inestable (Chávez, 2018). 
Así, frente a esta dificultad, determinar la estabilidad y la estabilidad asintótica de los puntos críticos es un problema delicado, ya que no disponemos de ningún medio para determinar la estabilidad, salvo que hallemos efectivamente todas las soluciones del sistema autónomo lineal, lo que puede resultar difícil, incluso, imposible.

Se investigó que estabilidad y estabilidad asintótica de un punto crítico del sistema dinámico autónomo lineal de coeficiente constante de la forma:

$$
\dot{\overline{\mathrm{x}}}=\mathrm{A}(\overline{\mathrm{x}})
$$

, donde A es una matriz cuadrada real no singular, se puede determinar utilizando el método de los valores propios y vectores propios.

Este artículo sobre la estabilidad tiene importancia en la moderna teoría de control y en la estabilidad de los sistemas dinámicos no lineales, que han sido investigados por matemáticos como Poincaré (1986), Liapunov (1942), Pontryagin (1980), Krasnov (1978), La Salle (1973), Mello (1979), Peixoto (1978), Kleider (1978), Sotomayor (1979) y otros.

\section{MARCO TEÓRICO}

\section{Definición (Punto crítico)}

Un punto $\overline{x^{*}} \in \mathrm{U}$, se denomina punto crítico del sistema dinámico autónomo lineal $(1,1)$ si $\mathrm{f}(\bar{x})=0$ (Sotomayor, 1979).

\section{Definición (Estabilidad)}

Un punto crítico $\overline{x^{*}}$ del sistema dinámico autónomo lineal $(1,1)$ es estable, cuando para esfera $U_{\varepsilon}$ de $\overline{x^{*}}$ en $U$ existe una esfera $U_{\delta}$ de $\overline{x^{*}}$ tal que toda solución $x(t)$ de $(1,1)$ con $\bar{x}(0) \in U_{\delta}$ está Definida en $U_{\varepsilon}, \forall t \geq 0$. Se representan en la figura 1 (Hirsh, 1978).

\section{Definición (Estabilidad asintótica)}

Un punto crítico $\overline{x^{*}}$ del sistema dinámico autónomo lineal $(1,1)$ es asintóticamente estable si $\overline{x^{*}}$ es estable y existe una vecindad $\mathrm{U}_{\delta_{1}}\left(\overline{x^{*}}\right) \subset \mathrm{U}_{\delta}\left(\overline{x^{*}}\right)$, tal que $\lim _{t \rightarrow \infty} x(t)=\overline{x^{*}}$. Se representan en la figura 1 (Kleider, 1978).

\section{Definición (Inestabilidad)}

Un punto crítico $\overline{x^{*}}$ del sistema dinámico autónomo lineal $(1,1)$ que no es estable se llama inestable. Tal como se muestra en la figura 1 (Kleider, 1978).

\section{Figura 1}

Estabilidad de los puntos críticos

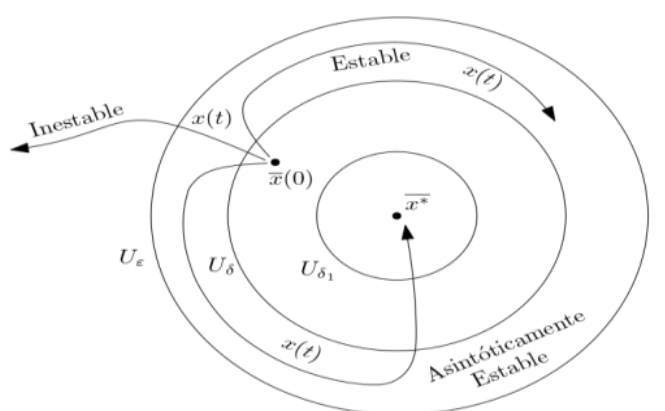

Fuente: Elaboración propia con software IPE 


\section{Definición (Autovector y Autovalor)}

Sea $A \in M_{n}(\mathbb{R}), v \in \mathbb{R}^{n}$ es un autovector (o vector propio) de A si $v \neq \mathbf{0}$ y $\exists \lambda \in \mathbb{R}$ talque $A v=\lambda v, \lambda$ se denomina autovalor (o valor propio) de A asociado a $v$.

\section{Proposición (Vector propio)}

Cada valor propio tiene asociado muchos vectores propios.

\section{Prueba}

Si $\boldsymbol{\lambda}$ es un valor propio asociado al vector propio $\mathbf{v}$, entonces $\mathbf{c v}$ es también un vector propio asociado a $\lambda$, para todo escalar $\mathbf{c}$. En efecto,

$$
\mathrm{A}(\mathrm{cv})=\mathrm{cAv}=\mathrm{c} \lambda \mathrm{v}=\lambda(\mathrm{cv})
$$

\section{Definición (Ecuación característica)}

Si $A \in M_{n}(\mathbb{R})$ y $\lambda$ es un valor propio de $A$ entonces $\operatorname{det}(A-\lambda I)=0$, se denomina ecuación característica de A.

\section{Definición (Polinomio Característico)}

Sea $A \in M_{n}(\mathbb{K})$. El polinomio característico de $A$ se $\operatorname{define~} \operatorname{como} \operatorname{det}(t I-A)$, es decir,

$$
P_{A}(\mathrm{t})=\operatorname{det}(t I-A)
$$

Proposición (Matriz triangular y valores propios)

Si $A \in M_{n}(\mathbb{R})$ es una matriz triangular superior e inferior, entonces los valores propios de A son los elementos de la diagonal principal.

\section{Prueba}

Sea $\left(\begin{array}{ccc}a_{11} & \cdots & 0 \\ \vdots & \ddots & \vdots \\ a_{n 1} & \cdots & a_{n n}\end{array}\right)$, entonces

$$
\begin{gathered}
(\lambda \mathrm{I}-\mathrm{A})=\left(\begin{array}{ccc}
\lambda-a_{11} & \cdots & 0 \\
\vdots & \ddots & \vdots \\
a_{n 1} & \cdots & \lambda-a_{n n}
\end{array}\right) \\
p(\lambda)=\operatorname{det}(A-\lambda I)=\left(\lambda-a_{11}\right) \ldots\left(\lambda-a_{n n}\right)
\end{gathered}
$$

Luego, $p(\lambda)=0 \Leftrightarrow \lambda=a_{i i}, \forall i=\overline{1, n}$

\section{Proposición (Valor propio)}

Sea $A \in M_{n}(\mathbb{K}), \lambda \in \mathbb{K}$ es un valor propio de A si y solo si $P_{A}(\mathrm{t})=0$

\section{Prueba}

Sea $\lambda \in \mathbb{K}$ es un valor propio de $\mathrm{A} \Rightarrow \mathrm{A}-\lambda \mathrm{I}$ no es invertible $\Rightarrow \operatorname{det}(\mathrm{A}-\lambda \mathrm{I})=$ $0 \Leftrightarrow P_{A}(\lambda)=0$

\section{Proposición (Matriz de cambio de base)}

Sean $A$ y $B \in \mathrm{M}_{\mathrm{n}}(\mathbb{K})$ tal que $\mathrm{B}$ es invertible entonces $P_{A}(\mathrm{t})=P_{B^{-1} A B}(\mathrm{t})$

\section{Prueba}

$$
\begin{gathered}
\mathrm{P}_{\mathrm{A}}(\mathrm{t})=\operatorname{det}(\mathrm{tI}-\mathrm{A})=\operatorname{det}\left(\mathrm{BB}^{-1}(\mathrm{tI}-\mathrm{A})\right. \\
=\mathrm{P}_{\mathrm{B}^{-1} \mathrm{AB}}(\mathrm{t})
\end{gathered}
$$




\section{Definición (Matriz diagonalizable)}

Una matriz $A \in M_{n}(\mathbb{R})$ es diagonalizable si es semejante una matriz diagonal J, es decir, existe una matriz invertible tal que $\mathrm{J}=\mathrm{X}^{-1} \mathrm{AX}$

\section{MATERIAL Y MÉTODOS}

En el presente artículo se consideró como material de estudio un sistema dinámico autónomo lineal de coeficientes constantes, es decir, un sistema de la forma:

$$
\dot{\overline{\mathrm{x}}}=\mathrm{A}(\overline{\mathrm{x}})
$$

, donde A es una matriz cuadrada real y el origen es el único punto crítico, cuando 0 no es un valor propio de A, es decir, A es una matriz no singular.

El método utilizado fue inductivo-deductivo, que permitió establecer un conjunto de enunciados a partir de los cuales y por reglas de inferencia lógicas se derivaron otras proposiciones (Guardales, 2004).

\section{RESULTADOS}

\section{Estabilidad Asintótica de un sistema dinámico autónomo lineal homogéneo:}

\section{Proposición (Asintóticamente estable)}

Si $\dot{\bar{x}}=A \bar{x}$ es un sistema dinámico lineal homogéneo cuya matriz de coeficientes $A \in$ $\mathrm{M}_{\mathrm{n}}(\mathbb{R})$ es no singular, entonces el punto crítico $\overline{0} \in \mathbb{R}^{n}$ es asintóticamente estable si las partes reales de todos los valores propios de A son negativos.

\section{Prueba}

Sea $\dot{\overline{\mathrm{X}}}=\mathrm{A} \bar{x}$ un sistema autónomo en la que sus soluciones pueden escribirse cuando las partes de todos los valores propios y distintos de A son negativos, es decir, $\lambda_{1}<$ $0, \ldots, \lambda_{n}<0$ y el sistema $\dot{\overline{\mathrm{x}}}=\mathrm{A} \bar{x}$, tiene $\mathrm{n}$ soluciones linealmente independientes construidas a partir de funciones de la forma $V_{\lambda_{i}} \mathrm{e}^{\lambda_{i} \mathrm{t}} k_{i}$, donde $\lambda_{i}, \forall i=\overline{1, n}$ valores propios, $V_{\lambda_{i}}, \forall i=\overline{1, n}$ los vectores propios asociados a los $\lambda_{i} \mathrm{y} k_{i}, \forall i=\overline{1, n}$ constante arbitrarias. Supongamos que $t \geq 0$, entonces $\left\|V_{\lambda_{i}} \mathrm{e}^{\lambda_{i} \mathrm{t}} k_{i}\right\|=\left\|V_{\lambda_{i}}\right\| \mathrm{e}^{\lambda_{i} \mathrm{t}}\left|k_{i}\right|, \quad$ y como $\left\|V_{\lambda_{i}}\right\| \geq 0$, tenemos que, si $\lambda_{i}<0$ entonces $\lim _{\mathrm{t} \rightarrow \infty}\left\|V_{\lambda_{i}} \mathrm{e}^{\lambda_{i} \mathrm{t}}\right\|=0$. Pero si $\lambda_{i}>$ 0 entonces $\left\|V_{\lambda_{i}} \mathrm{e}^{\lambda_{i} \mathrm{t}}\right\|$, no está acotada. Por lo tanto, toda trayectoria de $\dot{\overline{\mathrm{x}}}=\mathrm{A}(\overline{\mathrm{x}})$, que se origina en todos los valores propios reales y distintos para la matriz no singular A, se aproximan al punto crítico $\overline{0} \in \mathbb{R}^{n}$ cuando $t \rightarrow+\infty$, si la parte real de todos los valores propios es negativa, por tanto, el punto crítico es asintóticamente estable, y se alejará del origen si la parte real de todos los valores propios es positiva, por tanto, el punto crítico es inestable.

\section{Estabilidad de un sistema dinámico autónomo:}

\section{Proposición (Estable)}

Si $\dot{\overline{\mathrm{X}}}=\mathrm{A} \bar{x}$ es un sistema dinámico lineal homogéneo cuya matriz de coeficientes $\mathrm{A} \in$ $\mathrm{M}_{\mathrm{n}}(\mathbb{R})$ es no singular, entonces el punto crítico $\overline{0} \in \mathbb{R}^{n}$ es estable, si A tiene un par de valores propios imaginarios puros de multiplicidad uno, ningún par de valores propios imaginarios de multiplicidad mayor que uno, y ningún valor propio con partes reales positivas.

\section{Prueba}


Sea $\dot{\overline{\mathrm{X}}}=\mathrm{A} \bar{x}$ un sistema autónomo, entonces sus soluciones pueden escribirse como $\alpha+$ $i \beta, \beta>0$ con valores propios complejos de multiplicidad $m$ y además tiene $2 \mathrm{~m}$ soluciones linealmente independientes construidas a partir de funciones de la forma $V_{1 \lambda} \mathrm{e}^{\alpha \mathrm{t}} \mathrm{t}^{\mathrm{k}} \cos (\beta \mathrm{t})$, y $V_{2 \lambda} \mathrm{e}^{\alpha \mathrm{t}} \mathrm{t}^{\mathrm{k}} \operatorname{sen}(\beta \mathrm{t})$, donde $\mathrm{k}$ es un entero que toma los valores de $\overline{0,(m-1)}, V_{1 \lambda}, V_{2 \lambda}$ son vectores propios de A. Supongamos que $t \geq 0$, entonces

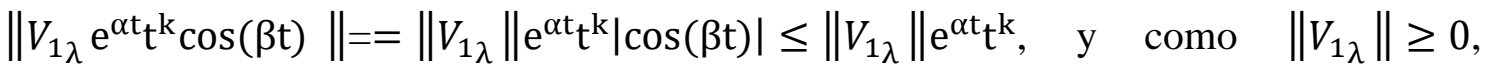
tenemos que, si $\alpha<0$ entonces $\lim _{\mathrm{t} \rightarrow \infty}\left\|V_{1 \lambda} \mathrm{e}^{\alpha \mathrm{t} \mathrm{t}^{\mathrm{k}}} \cos (\beta \mathrm{t})\right\|=0$. Pero, si $\alpha>$ 0 entonces $\left\|V_{1 \lambda} \mathrm{e}^{\alpha \mathrm{t}^{\mathrm{k}}} \cos (\beta \mathrm{t})\right\|$ no está acotado.

De igual manera, para la otra función si $\alpha<0$ entonces, $\lim _{\mathrm{t} \rightarrow \infty}\left\|\mathrm{V}_{2 \lambda} \mathrm{e}^{\alpha \mathrm{t}} \mathrm{t}^{\mathrm{k}} \operatorname{sen}(\beta \mathrm{t})\right\|=$ 0 Pero, si $\alpha>0$ entonces, $\left\|\mathrm{V}_{2 \lambda} \mathrm{e}^{\alpha \mathrm{t}^{\mathrm{k}}} \operatorname{sen}(\beta \mathrm{t})\right\|$ no está acotado.

De todo esto, tenemos que toda trayectoria de un sistema autónomo homogéneo, que se origina de un par de valores propios complejos conjugados para la matriz no singular A, se aproximan al punto crítico $\overline{0} \in \mathbb{R}^{n}$ cuando $t \rightarrow \infty$, si la parte real de estos valores propios es negativa, por tanto, el punto crítico es asintóticamente estable, y se apartará arbitrariamente del origen si la parte real de estos valores propios es positiva, por tanto, el punto es inestable.

En el caso que la matriz no singular admita un par de valores propios imaginarios puros $\pm i \beta$, si estos valores propios tienen multiplicidad 1, las soluciones correspondientes del sistema $\dot{\bar{x}}=\mathrm{A} \bar{x}$ se construyen de las funciones $V_{1 \lambda} \cos (\beta \mathrm{t})$, y $V_{2 \lambda} \operatorname{sen}(\beta \mathrm{t})$, y sabiendo que $\cos (\beta \mathrm{t})$ y $\operatorname{sen}(\beta \mathrm{t})$, están acotados cuando $t \rightarrow \infty$ implican que las trayectorias tienen las propiedades que requieren para la estabilidad, pero no para la estabilidad asintótica.

Ahora, si la multiplicidad de $\pm i \beta$ es mayor que 1 , el sistema $\dot{\overline{\mathrm{x}}}=\mathrm{A} \bar{x}$ admite soluciones en la que intervienen las funciones $V_{1 \lambda} \mathrm{t}^{\mathrm{k}} \cos (\beta \mathrm{t})$ y $V_{2 \lambda} \mathrm{t}^{\mathrm{k}} \operatorname{sen}(\beta \mathrm{t}) \quad \operatorname{con} \mathrm{k}>1 . \mathrm{v}$

Cuando esto suceda el origen es inestable, ya que $\left|\mathrm{t}^{\mathrm{k}} \cos (\beta \mathrm{t})\right| \mathrm{y}\left|\mathrm{t}^{\mathrm{k}} \mathrm{en}(\beta \mathrm{t})\right|$, no son acotados.

\section{DISCUSIÓN}

Consideremos un sistema dinámico lineal homogéneo,

$\left\{\begin{array}{l}\dot{x}=a x+b y \\ \dot{y}=c x+d y\end{array}\right.$

La hipótesis de que A es una matriz no singular implica que la matriz $A=\left[\begin{array}{ll}a & b \\ c & d\end{array}\right]$ es invertible. Luego, el origen es el único punto crítico de $(1,3)$.

Distinguiremos los siguientes casos:

\section{Matriz A con valores propios reales y distintos:}

Si la matriz A tiene valores propios reales y distintos $\lambda_{1} y \lambda_{2}$ del sistema $\dot{\bar{x}}=A \bar{x}$ tal que existe una matriz invertible $B$ entonces el nuevo sistema es $\dot{\bar{z}}=J \bar{z}$, donde $J=\left[\begin{array}{cc}\lambda_{1} & 0 \\ 0 & \lambda_{2}\end{array}\right]$ En efecto, supongamos que $\mathrm{v}_{1} \mathrm{y} \mathrm{v}_{2}$ son los vectores propios asociados a $\lambda_{1} \mathrm{y} \lambda_{2}$, entonces $B=\left\{v_{1}, v_{2}\right\}$ es una matriz de cambio de base. Luego, obtenemos la matriz de cambio de coordenadas $\overline{\mathrm{x}}=\mathrm{B} \overline{\mathrm{z}}$, es decir la ecuación se transforma 


$$
\overline{\mathrm{z}}=\mathrm{B}^{-1} \overline{\mathrm{x}} \Rightarrow \dot{\bar{z}}=B^{-1} \dot{\bar{x}}=B^{-1} A \bar{x}=B^{-1} A B \bar{z}=\mathrm{J} \overline{\mathrm{z}}
$$

Por lo tanto $\dot{\overline{\mathrm{Z}}}=\mathrm{J} \overline{\mathrm{Z}}$

En la nueva base $\left\{v_{1}, v_{2}\right\}$ el sistema tiene la siguiente forma

$\left\{\begin{array}{l}\dot{z_{1}}=\lambda_{1} z_{1} \\ \dot{z_{2}}=\lambda_{2} z_{2}\end{array}\right.$

Haciendo $k_{1}=k_{2}=0$ en las curvas de fase $\bar{\alpha}(t)=\left(k_{1} e^{\lambda_{1} t}, k_{2} e^{\lambda_{2} t}\right)$, se obtiene el punto crítico $(0,0)$.

Luego, se tiene los subcasos:

i) Si $\lambda_{2}<\lambda_{1}<0$ y $k_{1}=0, k_{2} \neq 0$ se obtiene dos semirrectas, en que $\mathrm{z}_{2} \rightarrow 0$ si $\rightarrow \infty$ y también si $k_{1} \neq 0, k_{2}=0$ se obtiene dos semirrectas, en el que $z_{1} \rightarrow 0$ si $t \rightarrow \infty$. Por lo tanto, el punto crítico $(0,0)$ es un nodo asintóticamente estable. Las órbitas son parábolas en el nuevo sistema tal como se muestra en la Figura 2.

\section{Figura 2}

Es un punto asintóticamente estable

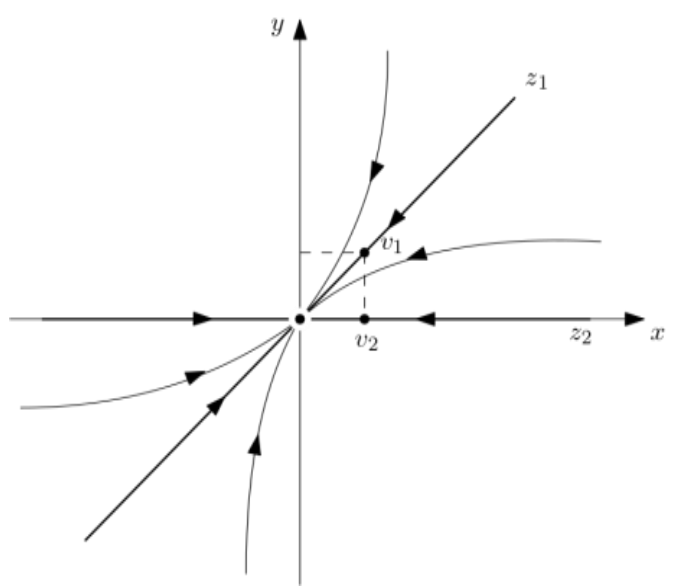

Fuente: Elaboración propia con software IPE

ii) Si $\lambda_{1}>\lambda_{2}>0$ y $k_{1}=0, k_{2} \neq 0$ se obtiene dos semirrectas, en el que $\mathrm{z}_{2} \rightarrow \infty$ si $\mathrm{t} \rightarrow$ $\infty$ y además $k_{1} \neq 0$,

$k_{2}=0$ se obtiene dos semirrectas, en el que $z_{1} \rightarrow \infty$ si $t \rightarrow \infty$. Por lo tanto, el punto crítico $(0,0)$ es un nodo inestable.

Las órbitas son parábolas en el nuevo sistema tal como se muestra en la figura 3.

\section{Figura 3}

Es un punto asintóticamente estable 


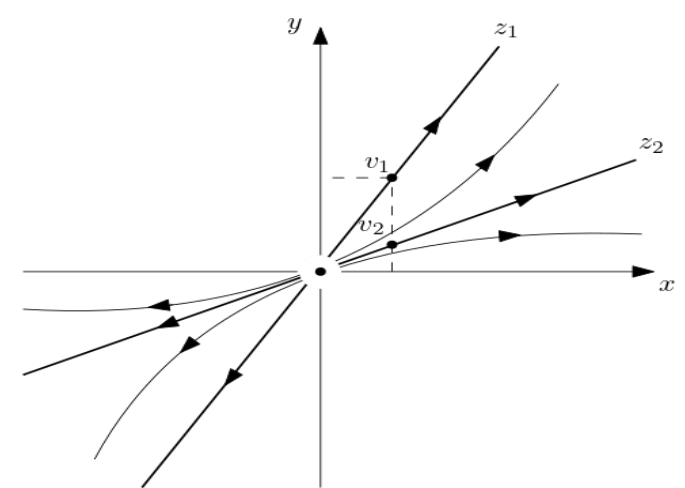

Fuente: Elaboración propia con software IPE

iii) Si $\lambda_{2}>0<\lambda_{1}$ y $k_{1}=0, k_{2} \neq 0$ se obtiene dos semirrectas, en que $\mathrm{z}_{2} \rightarrow \infty$ si $\mathrm{t} \rightarrow$ $\infty$, y además si $k_{1} \neq 0 \quad, k_{2}=0$ se obtiene dos semirrectas, en el que $z_{1} \rightarrow 0$ si $t \rightarrow$ $\infty$. Por lo tanto, el punto crítico $(0,0)$ es un punto silla. Las órbitas son hipérbolas en el nuevo sistema tal como se muestra en la figura 4.

\section{Figura 4}

\section{Es un punto silla}

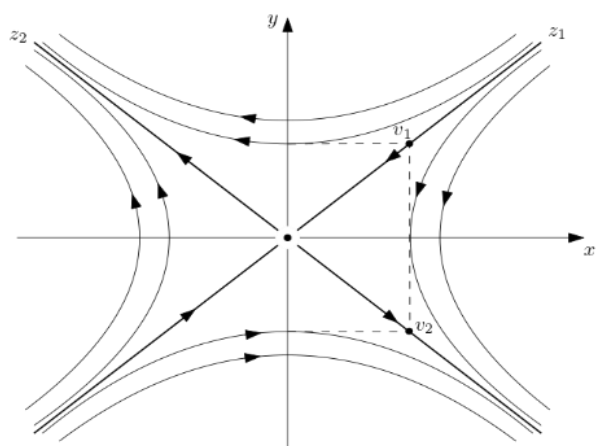

Fuente: Elaboración propia con software IPE

\section{Matriz diagonal A con valores propios reales e iguales:}

Si la matriz diagonal A tiene valores propios reales e iguales $\lambda_{1}=\lambda_{2}=\lambda$ del sistema $\dot{\overline{\mathrm{X}}}=\mathrm{A} \bar{x}$ tal que existe una matriz invertible $\mathrm{B}$ entonces el nuevo sistema es $\dot{\overline{\mathrm{Z}}}=\mathrm{J} \bar{z}$, donde $\mathrm{J}=\left[\begin{array}{ll}\lambda & 0 \\ 0 & \lambda\end{array}\right]$

En efecto, supongamos que $\mathrm{v}_{1}$ es el vector propio asociado a $\lambda$ y $\mathrm{v}_{2}$ es el vector propio degenerado asociados $\lambda$, entonces $B=\left\{v_{1}, v_{2}\right\}$ es una matriz de cambio de base. Luego, obtenemos la matriz de cambio de coordenadas

$\overline{\mathrm{x}}=\mathrm{B} \overline{\mathrm{Z}}$, es decir la ecuación se transforma

$$
\overline{\mathrm{z}}=\mathrm{B}^{-1} \overline{\mathrm{x}} \Rightarrow \dot{\overline{\mathrm{z}}}=B^{-1} \dot{\bar{x}}=B^{-1} A \bar{x}=B^{-1} A B \overline{\mathrm{z}}=\mathrm{J} \overline{\mathrm{Z}}
$$

Por lo tanto $\dot{\overline{\mathrm{Z}}}=\mathrm{J} \overline{\mathrm{Z}}$

En la nueva base $\left\{v_{1}, v_{2}\right\}$ el sistema se tiene la siguiente forma $\left\{\begin{array}{l}\dot{z_{1}}=\lambda z_{1} \\ \dot{z_{2}}=\lambda z_{2}\end{array}\right.$

Resolviendo el sistema se obtiene $\mathrm{z}_{2}=\mathrm{cz}_{1}$. Las órbitas son rectas en el nuevo sistema. Haciendo $k_{1}=k_{2}=0$ en las curvas de fase $\bar{\alpha}(t)=\left(k_{1} e^{\lambda t}, k_{2} e^{\lambda t}\right)$, se obtiene el punto crítico $(0,0)$. 
Luego, se tiene los subcasos:

i) Si $\lambda<0 y k_{1}=0, k_{2} \neq 0$ se obtiene dos semirrectas, en el que $\mathrm{z}_{2} \rightarrow 0$ si $\mathrm{t} \rightarrow \infty$.Ademas, $\lambda<0, k_{1} \neq 0, k_{2}=0$ se obtiene dos semirrectas, en el que $z_{1} \rightarrow$ 0 si $t \rightarrow \infty$. Por lo tanto, el punto $(0,0)$ es un nodo estelar asintóticamente estable tal como se muestra en la figura 5.

\section{Figura 5}

Nodo estelar asintóticamente estable

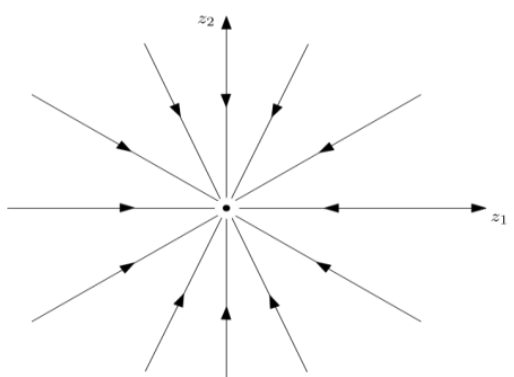

Fuente: Elaboración propia con software IPE

ii) De igual manera, si $\lambda>0, k_{1}=0, k_{2} \neq 0$ se obtiene dos semirrectas, en el que $z_{2} \rightarrow$ $\infty$ si $\mathrm{t} \rightarrow \infty$. Luego, si $\lambda>0, k_{1} \neq 0, k_{2}=0$ tenemos dos semirrectas, en el que $z_{1} \rightarrow$ $\infty$ si $t \rightarrow \infty$. Por lo tanto, el punto $(0,0)$ es un nodo estelar inestable tal como se muestra en la figura 6.

\section{Figura 6}

Nodo estelar inestable

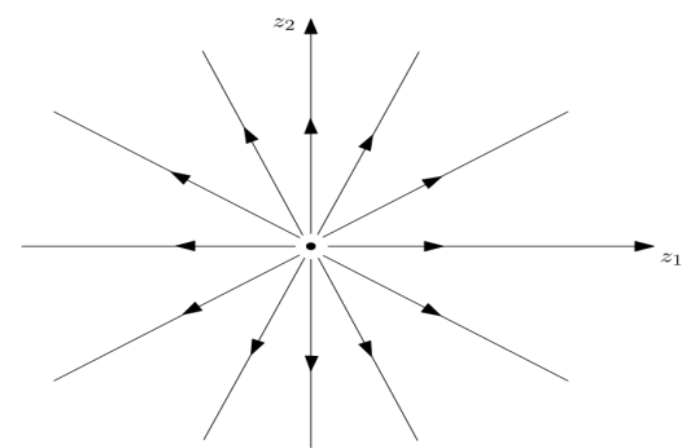

Fuente: Elaboración propia con software IPE

\section{Matriz no diagonal A con valores propios reales e iguales}

Si la matriz A no es diagonal y tiene valores propios reales e iguales $\lambda_{1}=\lambda_{2}=\lambda$ del sistema $\dot{\overline{\mathrm{X}}}=\mathrm{A} \bar{x}$ tal que existe una matriz invertible $\mathrm{B}$ entonces, el nuevo sistema es $\dot{\overline{\mathrm{Z}}}=$ $\mathrm{J} \bar{z}$, donde $\mathrm{J}=\left[\begin{array}{ll}\lambda & 1 \\ 0 & \lambda\end{array}\right]$. En efecto, supongamos que $\mathrm{v}_{1}$ es el vector propio asociado a $\lambda \mathrm{y}$ $\mathrm{v}_{2}$ es el vector propio degenerado asociados $\lambda$, entonces $B=\left\{v_{1}, v_{2}\right\}$ es una matriz de cambio de base. Luego, obtenemos la matriz de cambio de coordenadas $\overline{\mathrm{x}}=\mathrm{B} \overline{\mathrm{z}}$, es decir la ecuación se transforma

$\overline{\mathrm{z}}=\mathrm{B}^{-1} \overline{\mathrm{x}} \Rightarrow \dot{\bar{z}}=B^{-1} \dot{\bar{x}}=B^{-1} A \bar{x}$

Por lo tanto $\dot{\overline{\mathrm{Z}}}=\mathrm{J} \overline{\mathrm{Z}}$

$$
=B^{-1} A B \bar{z}=\mathrm{J} \overline{\mathrm{Z}}
$$


En la nueva base $\left\{v_{1}, v_{2}\right\}$ el sistema tiene la siguiente forma

$$
\left\{\begin{array}{c}
\dot{z}_{1}=\lambda z_{1}+z_{2} \\
\dot{z}_{2}=\lambda z_{2}
\end{array}\right.
$$

Haciendo $k_{1}=k_{2}=0$ en las curvas de fase $\bar{\alpha}(t)=\left(k_{1} e^{\lambda t} t+k_{2} e^{\lambda t}, k_{2} e^{\lambda t}\right)$, se obtiene el punto crítico $(0,0)$.

Luego, tenemos los subcasos:

i) Si $\lambda>0, k_{2}=0, k_{1} \neq 0$ se obtiene dos semirrectas, en el que $\mathrm{z}_{1} \rightarrow \infty$ si $\mathrm{t} \rightarrow$ $\infty$.Ademas si $\lambda>0, k_{2} \neq 0, k_{1}=0$ se obtiene dos semirrectas, en el que $\mathrm{z}_{2} \rightarrow$ $\infty$ si $\mathrm{t} \rightarrow \infty$. Luego,

$\frac{z_{1}}{z_{2}}=\frac{k_{1} t+k_{2}}{k_{2}} \rightarrow \infty$ si $t \rightarrow \infty$, las órbitas se apartan de $(0,0)$ tangencialmente al eje $z_{1}$. Por lo tanto, el punto $(0,0)$ es nodo degenerado inestable tal como se muestra en la figura 7 .

\section{Figura 7}

Nodo generado inestable

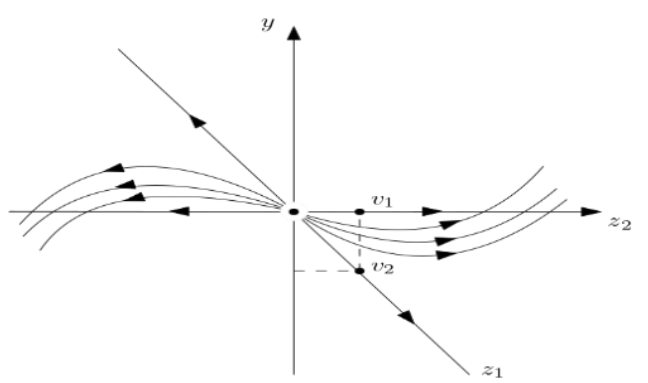

Fuente: Elaboración propia con software IPE

ii) Si $\lambda<0, k_{2}=0, k_{1} \neq 0$ se obtiene dos semirrectas, en el que $\mathrm{z}_{1} \rightarrow 0$ si $\mathrm{t} \rightarrow \infty$.

Además, si $\lambda<0, k_{2} \neq 0, k_{1}=0$ se obtiene dos semirrectas, en el que $\mathrm{z}_{2} \rightarrow 0$ si $\mathrm{t} \rightarrow$ $\infty$

$\frac{z_{2}}{z_{1}}=\frac{k_{2}}{k_{1} t+k_{2}} \rightarrow 0$ si $\mathrm{t} \rightarrow \infty$, las órbitas se aproximan al $(0,0)$ tangencialmente con respecto al eje $z_{1}$.

Por lo tanto, el punto $(0,0)$ es nodo degenerado estable tal como se muestra en la figura 8.

\section{Figura 8}

Nodo degenerado estable 


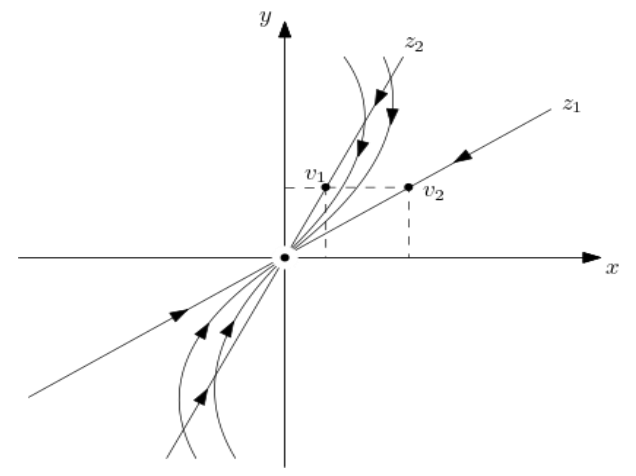

Fuente: Elaboración propia con software IPE

\section{Matriz diagonal A con valores propios compleja y distinta}

Si la matriz A tiene valores propios conjugados $\lambda_{1}=\alpha+i \beta$ y $\lambda_{2}=\alpha-i \beta$ del sistema $\dot{\overline{\mathrm{X}}}=\mathrm{A} \bar{x}$ entonces el nuevo sistema es $\dot{\overline{\mathrm{Z}}}=\mathrm{J} \bar{z}$, donde $\mathrm{J}=\left[\begin{array}{cc}\alpha & \beta \\ -\beta & \alpha\end{array}\right]$.

En efecto, supongamos que $v_{1}=\vec{a}+i \vec{b}, v_{2}=\vec{a}-i \vec{b}$ son los vectores propios asociados a los valores propios $\lambda_{1}$ y $\lambda_{2}$, donde $\vec{a}=\left(\begin{array}{l}a_{1} \\ a_{2}\end{array}\right)$, y $\vec{b}=\left(\begin{array}{l}b_{1} \\ b_{2}\end{array}\right)$. Luego, tenemos:

$$
A(\vec{a}+i \vec{b})=(\alpha+i \beta)(\vec{a}+i \vec{b})
$$

Por tanto, $\left\{\begin{array}{l}A \vec{a}=\alpha \vec{a}-\beta \vec{b} \\ A \vec{b}=\alpha \vec{b}+\beta \vec{a}\end{array}\right.$

Sea la matriz:

$$
\begin{gathered}
M=\left[\begin{array}{ll}
a_{1} & b_{1} \\
a_{2} & b_{2}
\end{array}\right] \Rightarrow A M=\left[\begin{array}{ll}
\alpha a_{1}-\beta b_{1} & \alpha b_{1}+\beta a_{1} \\
\alpha a_{2}-\beta b_{2} & \alpha b_{2}+\beta a_{2}
\end{array}\right] \\
, J=\left[\begin{array}{ll}
a & b \\
c & d
\end{array}\right] \Rightarrow M J=\left[\begin{array}{ll}
a_{1} a+b_{1} c & a_{1} b+b_{1} d \\
a_{2} a+b_{2} c & a_{2} b+b_{2} d
\end{array}\right]
\end{gathered}
$$

Igualando $\mathrm{AM}=\mathrm{MJ}$, tenemos:

$$
\mathrm{J}=\left[\begin{array}{ll}
a & b \\
c & d
\end{array}\right]=\left[\begin{array}{cc}
\alpha & \beta \\
-\beta & \alpha
\end{array}\right]
$$

En la nueva base $\left\{\mathrm{v}_{1}, \mathrm{v}_{2}\right\}$ el sistema se convierte $\dot{\overline{\mathrm{Z}}}=\mathrm{J} \overline{\mathrm{z}}$. Por ejemplo, resolver el sistema $\left\{\begin{array}{c}\dot{z_{1}}=\alpha z_{1}+\beta z_{2} \\ \dot{z_{2}}=-\beta z_{1}+\alpha z_{2}\end{array}\right.$

Luego, utilizando coordenadas polares

$\left\{\begin{array}{l}\mathrm{z}_{1}=\mathrm{r} \cos \theta \\ \mathrm{z}_{2}=\mathrm{r} \operatorname{sen} \theta\end{array} \Rightarrow \begin{array}{l}\dot{\mathrm{z}_{1}}=\dot{\mathrm{r}} \cos \theta-\mathrm{r} \operatorname{sen} \theta \\ \dot{\mathrm{z}_{2}}=\dot{\mathrm{r}} \operatorname{sen} \theta+\mathrm{r} \cos \theta\end{array}\right.$

Tenemos

$\left\{\begin{array}{c}\dot{\mathrm{r}}=\dot{z_{1}} \cos \theta+\dot{z_{2}} \operatorname{sen} \theta \\ \dot{\theta}=\frac{1}{r}\left(\dot{z_{2}} \cos \theta-\dot{z_{1}} \operatorname{sen} \theta\right)\end{array}\right.$ 
Finalmente, se obtiene

$$
\left\{\begin{array}{l}
\dot{r}=\alpha r \\
\dot{\theta}=-\beta
\end{array}\right.
$$

$\mathrm{Al}$ resolver el sistema tenemos:

$\mathrm{r}=k_{2} e^{k_{1} \theta}$. Las órbitas tienen forma logarítmica en el nuevo sistema. En las curvas de fase $\bar{\alpha}(t)=\left(r(0) e^{\alpha t}, \theta(0)-\beta t\right)$, si $\beta>0$,

$\theta \rightarrow-\infty$, cuando $t \rightarrow+\infty$, las órbitas giran una infinidad de veces alrededor del punto crítico, la dirección de crecimiento de t será las de las agujas del reloj. Ahora, si $\beta<$ $0, \theta \rightarrow+\infty$ cuando $t \rightarrow-\infty$, la dirección de crecimiento de $t$ es contraria a las agujas del reloj.

Ahora, se presentan los sub casos.

i) Si $\alpha=0$, entonces $r(t)=r(0)$. Las órbitas son circunferencias con centro en el origen. El punto crítico $(0,0)$ se denomina centro tal como se muestra en la figura 9.

\section{Figura 9}

Centro estable

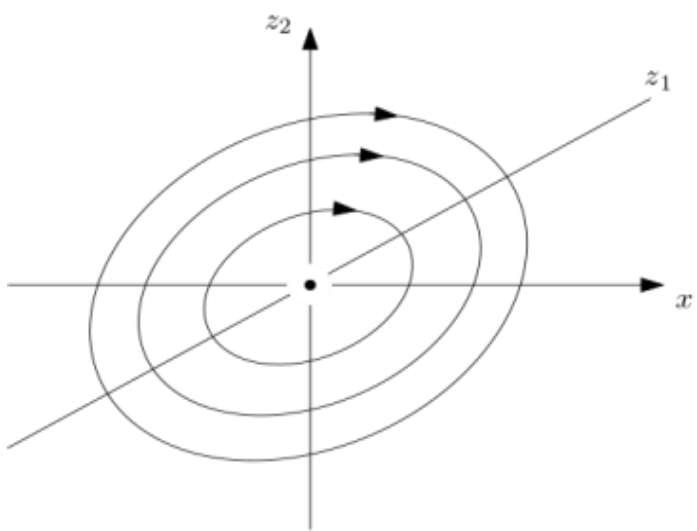

Fuente: Elaboración propia con software IPE

ii) Si $\alpha<0$, entonces $\mathrm{r}(\mathrm{t}) \rightarrow 0$ cuando $t \rightarrow+\infty$. Las órbitas son espirales logarítmicas. El punto crítico $(0,0)$ se denomina foco estable, tal como se muestra en la figura 10 .

\section{Figura 10}

Foco asintóticamente estable 


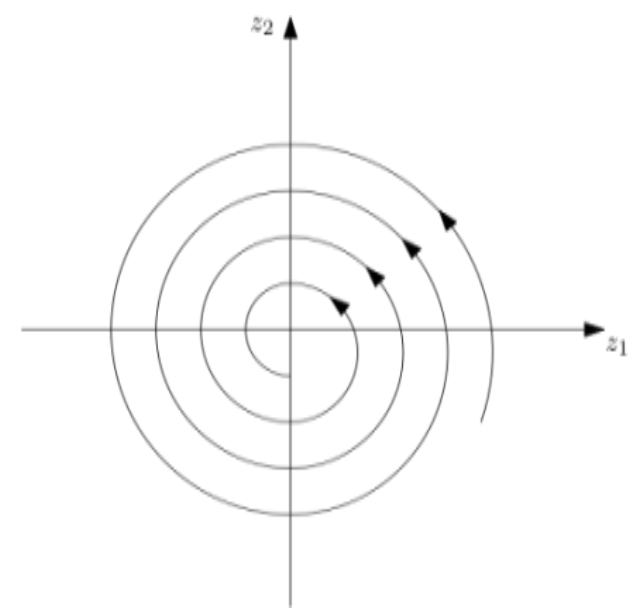

Fuente: Elaboración propia con software IPE

iii) Si $\alpha>0$, entonces

$r(t) \rightarrow+\infty$ cuando $t \rightarrow+\infty$. Las órbitas son espirales logarítmicas. El punto crítico $(0,0)$ se denomina foco estable tal como se muestra en la figura 11.

Figura 11

Foco inestable

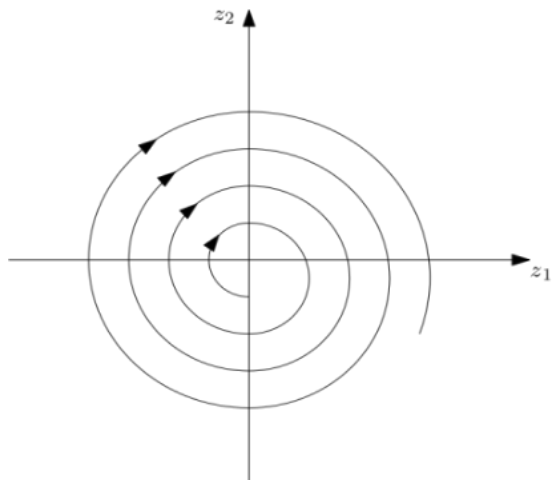

Fuente: Elaboración propia con software IPE

\section{CONCLUSIONES}

La estabilidad de los puntos críticos de un sistema autónomo lineal homogéneo es posible establecerlo al aplicar el método de los valores propios y vectores propios a una matriz no singular sin necesidad de resolver el sistema lineal homogéneo.

Si las partes reales de todos los valores propios de la matriz no singular son negativos entonces el punto crítico de un sistema lineal homogéneo es asintóticamente estable.

\section{REFERENCIAS}

Chávez, J., Rodríguez, E. \& Méndez, L. (2018). Criterio de estabilidad y estabilidad asintótica de la solución en los sistemas de ecuaciones diferenciales no lineales. Ciencias, 2 (1), 52-58.

Elsgolts, L. (1979). Ecuaciones Diferenciales y Cálculo Variacional. Mir, Moscú. 
Guardal, Roger. (2004). Investigación y enseñanza de la matemática: Sociedad de la matemática peruana. Primera ed. Perú.

Hirsh, M. (1983). Ecuaciones Diferenciales, Sistemas Dinámico y Álgebra Lineal. Alianza Editorial, Madrid.

Kreider, D. (1978). Ecuaciones Diferenciales. Fondo educativo interamericano, México. Krasnov, L. (1978). Teoría de la Estabilidad. Reverte, Barcelona.

La Salle, J. \& Lefschetz, S. (1973). Stability by Liapunov's Direct Method with Applications. Academic Press, New York.

Liapunov, A. (1949). Probleme General de la Stabilitie du Mouvement, Ann. of Math. Stud., Vol. 17. Princeton Univ. Press, Princeton, NJ.

Mello, A. (1979). Ecuaciones Diferenciales. Imeusp, Brasil.

Maxwell, J. (1868). On Governors. Proc. Roy. Soc. London, vol. 16, pp. 270-283.

Peixoto, M. (1978). Dynamical Systems. Academic Press, Nueva York.

Perko, L. (1991). Differential Equations and Dynamical Systems. Springer Verlag, New York.

Pontryagin, 1. (1980) Ecuaciones Diferenciales Ordinarias. Addison Werley, Reading, Masachussetts.

Poincaré, Russell, Zermelo et Peano. (1986) Textes de la discussion (1906-1912) sur les fondements des mathématiques: Des antinomies à la prédicativité. Edited by Gerhard Heinzmann. Paris: Blanchard.

Sotomayor, J. (1979). Ecuaciones Diferenciales Ordinarias, Euclides. Brasil. VPO. 